\title{
Problems of Mathematical Finance by Stochastic Control Methods ${ }^{\star}$
}

\author{
Łukasz Stettner \\ Institute of Mathematics Polish Academy of Sciences, Sniadeckich 8, 00-956 Warsaw and \\ Academy of Finance, Poland \\ stettner@impan.gov.pl
}

\begin{abstract}
The purpose of this paper is to present main ideas of mathematics of finance using the stochastic control methods. There is an interplay between stochastic control and mathematics of finance. On the one hand stochastic control is a powerful tool to study financial problems. On the other hand financial applications have stimulated development in several research subareas of stochastic control in the last two decades. We start with pricing of financial derivatives and modeling of asset prices, studying the conditions for the absence of arbitrage. Then we consider pricing of defaultable contingent claims. Investments in bonds lead us to the term structure modeling problems. Special attention is devoted to historical static portfolio analysis called Markowitz theory. We also briefly sketch dynamic portfolio problems using viscosity solutions to HamiltonJacobi-Bellman equation, martingale-convex analysis method or stochastic maximum principle together with backward stochastic differential equation. Finally, long time portfolio analysis for both risk neutral and risk sensitive functionals is introduced.
\end{abstract}

\section{Pricing of Financial Derivatives}

One of the fundamental problems of mathematics of finance is pricing of the derivative securities (shortly derivatives) i.e. securities the value of which depends on the basic securities such as stocks or bonds. In this section we restrict ourselves to stocks, although similar problems (unfortunately much harder) concern also derivatives of bonds.

\subsection{Modeling of Asset Prices}

We start with modeling of asset prices (stocks). We assume that we are given $d$ assets on the market and denote the price of the $i$-th asset at time $t$ by $S_{i}(t)$. We shall consider in parallel way two approaches: in discrete and in continuous time. We assume a given probability space $\left(\Omega, \mathscr{F},\left(\mathscr{F}_{t}\right), P\right)$. In the case of discrete time the asset prices satisfy the relation

$$
\frac{S_{i}(t+1)}{S_{i}(t)}=\zeta_{i}(t, z(t+1), \xi(t+1)),
$$

\footnotetext{
${ }^{\star}$ Research supported by MNiSzW grant no. 1 P03A 01328.
} 
for $i=1,2, \ldots, d$, where $(z(t))$ stands for the process of economic factors which have an impact on the prices, and $(\xi(t))$ is a noise process being a sequence of i.i.d. random variables such that $\xi(t)$ is independent of $z(s)$ for $s \leq t$.

In the case of continuous time model we assume that $S_{i}(t)$ is given by the following formula

$$
S_{i}(t)=S_{i}(0) e^{\int_{0}^{t} a_{i}(s, z(s)) d s+\int_{0}^{t} \sigma_{i}(s, z(s)) \cdot d L(s)},
$$

where the last term is the stochastic integral with respect to the process with stationary independent increments which is a Levy process or more specifically a Wiener process $L$. For the definition of stochastic integral we refer to [37].

\subsection{Contingent Claims}

A typical situation we encounter on financial markets is that at a given time $T$, called maturity we get a contingent claim the value of which is an $\mathscr{F}_{T}$ measurable random variable $H$. The problem is to determine its price at time 0 . European options can serve as an example of contingent claims. Assume that we want to have a guarantee that at time $T$ we shall buy one $i$-asset for the value of at most $K$ (the so called striking price). For this purpose we buy a European call option. If the price $S_{i}(T)$ is greater than $K$ we exercise this option buying one $i$-th asset for the price $K$. Otherwise, i.e. when $S_{i}(T)<K$ there is no reason to exercise this option - we simply buy the asset for the price $S_{i}(T)$. Consequently the value of this European call option is $\left(S_{i}(T)-K\right)^{+}$. Similarly, when we have an asset $i$ we can guarantee to sell it at time $T$ for the price $K$ buying a European put option. In this case the value of the option is $\left(K-S_{i}(T)\right)^{+}$. In both cases an important factor is the price for the option we have to pay at time 0 . The terminal maturity time $T$ is fixed and deterministic. One can consider contracts with random $T$. In the so called American options the buyer of an option is allowed to choose random maturity time $\tau$, i.e. he chooses the time to exercise the option. Clearly such call or put options are more expensive since they offer a better bargain to the buyer.

\subsection{Portfolio and Absence of Arbitrage}

From stochastic control point of view the main decision we make on financial markets is the choice of a proper investment portfolio. We denote by $V(0)=v$ the initial value of the wealth process. We assume that we can invest $v$ in the $d$-assets available on our market as well as in the savings account in a bank with a short term interest rate $r(t)$ at time $t$, which means that for one dollar invested at time 0 in the account we obtain $B_{t}=\exp \left(\int_{0}^{t} r_{u} d u\right)=S_{0}(t)$ at time $t$. An investment strategy at each time $t$ is a sequence $\left(N_{0}(t), N_{1}(t), \ldots, N_{d}(t)\right)$ where $N_{0}(t)$ is the capital invested in our savings account, and $N_{i}(t)$ is the capital invested in the $i$-th asset at time $t$. The strategy can be also described as the sequence $\pi=\left(\pi_{0}(t), \pi_{1}(t), \ldots, \pi_{d}(t)\right)$ where $\pi_{i}(t)$ is the portion of the wealth process $V(t)$ invested at time $t$ in the $i$-th asset (for $i=0$ in the savings account). Clearly $N_{i}(t)=\pi_{i}(t) V(t)$ for $i=0,1, \ldots, d$ and for discrete time model we have 


$$
\begin{aligned}
V(t) & =\sum_{j=0}^{d} N_{j}(t) S_{j}(t)=V(t-1)+\sum_{j=0}^{d} N_{j}(t-1)\left(S_{j}(t)-S_{j}(t-1)\right) \\
& =V(t-1) \sum_{j=0}^{d} \pi_{j}(t-1) \zeta_{j}(t, z(t), \xi(t)),
\end{aligned}
$$

with $\zeta_{0}(t, z, \xi)=\exp \left(\int_{t}^{t+1} r_{u} d u\right)$. The wealth process depends on the investment strategy $\pi$ and initial wealth $v$. To point out this we shall denote the wealth process at time $t$ by $V^{\pi, v}(t)$. We shall assume furthermore that the portfolio is selffinancing which means that no money is added or withdrawn i.e. we invest at each time $t$ the value equal to our wealth $V^{\pi, v}(t)$. The fundamental assumption of mathematical finance is the so called absence of arbitrage (AA) in time $T$, which is the absence of the existence of portfolio $\pi$ such that $V^{\pi, 0}(T) \geq 0$ and $P\left\{V^{\pi, 0}(T)>0\right\}>0$. Roughly speaking, it means that we are not able to create gain at time $T$ without risk. The economical notion of the absence of arbitrage has an important analytical interpretation, which is very transparent in discrete time case.

We call a probability measure $Q$, which is equivalent to the original probability measure $P$, a martingale measure, whenever $\left(\frac{S_{i}(t)}{B_{t}}\right)$ are martingales with respect to $Q$ for $i=1,2, \ldots, d$ and $t=0,1, \ldots, T$. The martingale property means integrability of the ratios $\frac{S_{i}(t)}{B_{t}}$ plus the property

$$
E\left\{\frac{S_{i}(t+1)}{B_{t+1}} \mid F_{t}\right\}=\frac{S_{i}(t)}{B_{t}},
$$

for $t=0,1, \ldots, T-1$. The following theorem (see [10] or [20]22] for more recent approaches) is fundamental in discrete time mathematics of finance.

Theorem 1. (Dalang, Morton, Willinger, 1990) The absence of arbitrage is equivalent to the existence of a martingale measure $Q$.

In what follows we shall denote by $\mathscr{Q}$ the family of all martingale measures. By Theorem 1 under (AA) we have $\mathscr{Q} \neq \emptyset$.

\subsection{Pricing}

We shall now consider the problem of pricing of contingent claim of value $H$ at a given maturity time $T$. The buyer of it collects a gain which is a random variable $H$. We would like to evaluate the price of $H$ at the initial time. One can look at this price from the perspective of the seller or the buyer. An acceptable price for the seller is such a price that for the amount he obtains at time 0 he is able (providing he invests it in an optimal way) to get at least the compensation for $H$ (i.e. to hedge $H$ ), which he is supposed to deliver to the buyer. This investment forms an optimal stochastic control problem. We would like to find the smallest initial capital $v$, which invested in an optimal way gives us at least the value $H$ at time $T$. Denote by $p_{s}(H)$ the minimal seller price. Consequently we can write the formula

$$
p_{s}(H)=\inf \left\{v: \exists_{\pi}, V^{\pi, v}(T) \geq H\right\} .
$$


The buyer price on the other hand is the value $v$ such that if he starts with initial capital $-v$ and invests it in an optimal way, then at time $T$ the value of his portfolio plus his gain $H$ is nonnegative. Such maximal $v$ forms the so called buyer price $p_{b}(H)$ and is the maximal price acceptable for the buyer. We have

$$
p_{b}(H)=\sup \left\{v: \exists_{\pi}, V^{\pi,-v}(T)+H \geq 0\right\} .
$$

Alternatively we can write that $p_{b}(H)=-p_{s}(-H)$. Under the absence of arbitrage assumption we clearly have that $p_{b}(H) \leq p_{s}(H)$. The interval $\left[p_{b}(H), p_{s}(H)\right]$ is called an absence of arbitrage interval and any price from this interval is acceptable in the sense that neither seller nor buyer is able to obtain a positive gain without risk at time $T$ (which is an arbitrage). In particular situations, when $p_{b}(H)=p_{s}(H)$ for all bounded $H$, we say that the market is complete which in turn corresponds to the fair price or fair game between the seller and the buyer. By Theorem 1 it is clear that in the case of complete market the family $\mathscr{Q}$ is a singleton.

Assuming integrability of the contingent claim with respect to the set of all martingale measures $\mathscr{Q}$, i.e. $\sup _{Q \in \mathscr{Q}} E^{Q}|H|<\infty$ we have the following analytic representations for the seller and buyer prices called the fundamental asset pricing theorem.

Theorem 2. Under (AA) we have

$$
p_{S}(H)=\sup _{Q \in \mathscr{Q}} E^{Q}\left\{\frac{H}{B_{T}}\right\},
$$

and

$$
p_{b}(H)=\inf _{Q \in \mathscr{Q}} E^{Q}\left\{\frac{H}{B_{T}}\right\} .
$$

The proof of this theorem (see [38]) is based on an important result from the theory of stochastic processes called optional decomposition.

Lemma 1. (Föllmer, Kabanov [16]) If $(Y(t))$ is a $Q$ supermartingale for each $Q \in$ $\mathscr{Q}$, there is $\pi$ and an adapted increasing process $\left(d_{t}\right), d_{0}=0$ such that $Y(T)=V^{\pi, Y(0)}$ $(T)-d_{T}$.

The absence of arbitrage interval $\left[p_{b}(H), p_{s}(H)\right]$ may be very large. Therefore one would like to find in this interval a proper price of the contingent claim. For this purpose we have to use other criteria: we choose martingale measures with minimal variance (see [38]) or minimal entropy (see [18]). Another approach is, instead of hedging with probability 1 , to consider the so called quantile hedging under which we require inequalities in (5) and (6) to be satisfied with certain probability, e.g. $1-\varepsilon$ (see [17]). Alternatively we can also use utility theory (see [18]) to price contingent claims. The theory described above was based on an assumption that there is no friction on our market, i.e. we do not pay transaction costs. The theory with proportional transaction costs (we pay costs proportional to the volume of transaction) is more complicated and still a subject of intensive studies (see e.g. [2165] and references therein). In the case of large transactions one can expect to pay even smaller proportional transaction costs. These considerations lead to concave transaction costs. The problems are very complicated 
and at this moment some results are only available for simple Cox-Ross-Rubinstein model introduced below in Sect. 1.6 (see [39] and [26]).

In what follows we tacitly assume that there are no restrictions on trade and we have a competitive market: trader can sell unlimited quantities of securities without changing the security's price. Clearly this is a serious simplification. There are two approaches to handle the liquidity risk caused by large transactions: either we assume that the transaction can change the asset price (see [7]) or the price remains unchanged but we consider convex transaction costs (see [8]).

The theory we introduced so far concerned financial derivatives which are exercised exactly at time $T$. The American type options can be exercised at any time $\tau$ from the interval $[0, T]$, which is chosen by the buyer of the option. Consequently a contingent claim is an $\left(\mathscr{F}_{t}\right)$ adapted process $H=\left(H_{t}\right)$. In particular $H_{t}=\left(\left(S_{1}(t)-K\right)^{+}\right)$or $H_{t}=\left(\left(K-S_{1}(t)\right)^{+}\right)$in the case of American call or put options. To avoid integrability problems assume that the process $H_{t}$ is bounded. One case easily adjust the formulae (5) and (6) for the seller $p_{s}(H)$ and for the buyer $p_{b}(H)$ prices (the inequalities should be satisfied for each $t$ from $[0, T]$, instead of $T$ only). By an analogy to Theorem 2 we have (see [18])

Theorem 3. Under (AA) we have

$$
p_{s}(H)=\sup _{Q \in \mathscr{Q}} \sup _{\tau} E^{Q}\left\{\frac{H_{\tau}}{B_{\tau}}\right\},
$$

and

$$
p_{b}(H)=\inf _{Q \in \mathscr{Q}} \sup _{\tau} E^{Q}\left\{\frac{H_{\tau}}{B_{\tau}}\right\},
$$

where $\sup _{\tau}$ denotes an optimal stopping problem: we maximize $E^{Q}\left\{\frac{H_{\tau}}{B_{\tau}}\right\}$ over all stopping times $\tau$.

\subsection{Continuous Time Markets}

In this section we consider the case, when asset prices $(S(t))$ have dynamics of the form (2) and consequently we are allowed to change our portfolio at any moment. In such a case we have our portfolio at time $t$ under the investment strategy $\pi$ by anology to (3) in the form

$$
V^{\pi, v}(t)=v+\int_{0}^{t} \pi(u) d S(u) .
$$

The notion of the absence of arbitrage (AA) remains the same. There is however a difference in the analytic form of the absence of arbitrage. First of all the notion of a martingale is replaced by a local martingale property. We say that $\frac{S_{i}(t)}{B_{t}}$ is a $Q$ local martingale if there is a sequence of stopping times $\tau_{n} \rightarrow \infty$ such that $\forall_{n}\left(\frac{S\left(t \wedge \tau_{n}\right)}{B_{t \wedge \tau_{n}}}\right)$ is a $Q$ martingale for $t \in[0, T]$. We shall denote by $\tilde{\mathscr{Q}}$ the set of all measures $Q$ equivalent to $P$ under which $\left(\frac{S(t)}{B_{t}}\right)$ is a local martingale.

Furthermore we introduce the so called no free lunch with vanishing risk property (NFLVR). It is satisfied when there is no sequence of strategies $\left(\pi^{k}(t)\right)$ such that 
- $\exists_{\alpha_{k}}, P\left\{V^{\pi^{k}, 0}(t) \geq \alpha_{k}, t \in[0, T]\right\}=1$,

- $\forall_{k}, \exists_{\delta_{1}, \delta_{2}>0}, P\left\{V^{\pi^{k}, 0}(T)>\delta_{1}\right\}>\delta_{2}$,

- $V^{\pi^{k}, 0}(T) \geq-\frac{1}{k}$.

We have (for the proof see [11])

Theorem 4. (Delbaen, Schachermayer, 1994)

$$
(A A) \Longleftarrow(N F L V R) \Longleftrightarrow \tilde{\mathscr{Q}} \neq \emptyset .
$$

Consequently we do not have full absence of arbitrage characterization as in discrete time.

\subsection{Complete Markets}

A particular situation arises, when $\mathscr{Q}$ or $\tilde{\mathscr{Q}}$ is a singleton. For discrete time model this is in the case when we have the so called Cox-Ross-Rubinstein model (CRR) (see [9]) consisting of the bank account $S_{0}(t)$ and an asset $S(t)$ with the dynamics

$$
\begin{gathered}
S_{0}(n+1)=(1+r) S_{0}(n), \\
S(n+1)=\left(1+\rho_{n}\right) S(n),
\end{gathered}
$$

where $r$ is deterministic and $\left(\rho_{n}\right)$ is a sequence of i.i.d. random variables taking values $u$ and $d$ only, where $d<r<u$.

Notice that in this case given $H$ which is $\mathscr{F}_{T}=\sigma\left\{\rho_{i}, i=0, \ldots, T-1\right\}$ measurable, there is an investment strategy $\pi=(\pi(t))$ and $v \in R$ such that $V^{\pi, v}(T)=H$, and consequently we have a replication of the contingent claim $H$. For other markets, say multinomial markets we have only hedging property i.e. there are $(\pi(t))$ and $v \in R$ such that $V^{\pi, v}(T) \geq H$.

In the case of a continuous time market, $\tilde{\mathscr{Q}}$ is a singleton when we have the so called Black and Scholes model (see [4]). We assume that the short term interest rate $r_{t}=r$ is deterministic and besides of the bank account there is one asset with the dynamics of its price $S(t)$ of the form

$$
S(t)=S(0) e^{\int_{0}^{t} a d s+\int_{0}^{t} \sigma d W(s)}
$$

where $a$ and $\sigma$ are deterministic. In this case we can obtain a famous analytic formula (called Black-Scholes formula) for the price of the European call option with the gain $H=(S(T)-K)^{+}$at time $T$. We have (see [4])

\section{Proposition 1}

$$
p_{s}(H)=p_{b}(H)=S(0) N\left(d_{1}(S(0), T)\right)-K e^{r T} N\left(d_{2}(S(0), T)\right),
$$

with $d_{1}(s, t)=\frac{\ln \frac{s}{R}+\left(r+0.5 \sigma^{2}\right) t}{\sigma \sqrt{t}}, d_{2}(s, t)=d_{1}(s, t)-\sigma \sqrt{t}$.

Notice that the formula does not depend on $a$, since the drift part $a t$ is hidden in the local martingale measure. 


\subsection{Stochastic Control Methods (Summary)}

We complete Sect. 1 with a summary of the stochastic control methods, used so far explicitly and implicitly. Generally speaking our main control problem was to find (minimal) $v$ such that $V^{\pi, v}(T) \geq H$ i.e. find a minimal initial capital under which we can hedge the contingent claim $H$. Absence of arbitrage problem can be also formulated in these terms. In discrete time case such control problem can be solved using backward dynamic programming (see e.g. [36]). In continuous time case situation is more complicated. This is a stochastic version of optimal tracking problem which can be solved using controlled Backward Stochastic Differential Equations (BSDEs) (see [13]). The problem with BSDEs stimulated purely analytical approach based on martingale method. Although we have a nice formula for the pricing problem, the evaluation of the seller or buyer price is a rather difficult computational problem. Another problem appears in pricing of American options. We have to solve an optimal stopping problem which in the case of large markets (when the number of assets $d$ is large) is not feasible.

\section{Credit Risk}

This section is devoted to special contingent claims which may default. In the previous section the contingent claim $H$ was delivered at time $T$, in the case of European options and its value was not a subject to any change. For a given time horizon $T$ a general defaultable contingent claim may be considered as a quintuple $(X, A, \tilde{X}, Z, \tau)$ consisting of the promised contingent claim $X$, promised dividends $\left(A_{t}\right)$ payed up to default time, recovery claim $\tilde{X}$ i.e. the value of contingent claim we obtain if we have default before $T$ and the recovery process $Z$, which is a certain recovery payoff at the time of default, payed when default occurs before or at the maturity $T$. We denote by $\tau$ default time, and by $H_{t}=1_{\tau \leq t}$ the so called default process. The dividend process of the defaultable contingent claim is of the form

$$
D_{t}=X^{d}(T) 1_{t \geq T}+\int_{j 0, t]}\left(1-H_{u}\right) d A_{u}+\int_{j 0, t]} Z_{u} d H_{u},
$$

with $X^{d}(T)=X 1_{\tau>T}+\tilde{X} 1_{\tau \leq T}$. The problem is to price such dividend process. There are two approaches which vary depending on the form of the default time. If $\tau$ is a stopping time with respect to the available observation of the market i.e. first entry time of the company wealth process to the so called bankruptcy region, we have a structural approach. In this approach default time is predictable with respect to the available observation. Consequently pricing of defaultable contingent claims leads to stochastic control problems which can be solved for particular form of $\tau$. An alternative approach called intensity based approach assumes that we are not able to predict $\tau$. Our observation at time $t$ is $\mathscr{G}_{t}=\mathscr{H}_{t} \vee \mathscr{F}_{t}$. We are not able to solve such problem using stochastic control methods. We consider therefore a martingale approach. It is not true in general that a $\left(\mathscr{G}_{t}\right)$ martingale measure is also an $\left(\mathscr{F}_{t}\right)$ martingale measure. To obtain an analytic formula we assume the so called invariance property which says that for a $\left(\mathscr{G}_{t}\right)$ martingale measure $Q^{*}$ : an $\left(\mathscr{F}_{t}\right)$ martingale is also a $\left(\mathscr{G}_{t}\right)$ martingale. Let $Q^{*}$ be a given $\left(\mathscr{G}_{t}\right)$ martingale measure. Under suitable integrability properties (see [2] for details) we have 
Theorem 5. The value of $D C C(X, A, 0, Z, \tau)$ at time $t$ is equal to

$$
1_{\tau>t} G_{t}^{-1} B_{t} E^{Q^{*}}\left\{\int_{t}^{T} B_{u}^{-1}\left(G_{u} d A_{u}-Z_{u} d G_{u}\right)+G_{T} B_{T}^{-1} X \mid \mathscr{F}_{t}\right\},
$$

with $G_{t}=P\left\{\tau>t \mid \mathscr{F}_{t}\right\}$.

\section{Term Structure Modeling}

In the theory developed in Sect.11we had an opportunity to invest in assets and savings account. We consider now investments in bonds also. By a zero coupon bond with maturity $T$ we mean a financial instrument paying to its holder one unit of cash at time $T$. Let $B(t, T)$ be the price of such bond at time $t \leq T$. We shall use the following representation of $B(t, T)$

$$
B(t, T):=\exp \left(-\int_{t}^{T} f(t, u) d u\right),
$$

where $f(t, T)$ is called an instantaneous forward rate. By the very definition clearly $B(T, T)=1$. Assume that $P^{*}$ is an equivalent measure to $P$ such that $\frac{B(t, T)}{B_{t}}$ is a $P^{*}$ martingale. From the martingale property we obtain

$$
B(t, T)=E^{P^{*}}\left\{e^{-\int_{t}^{T} r_{u} d u} \mid F_{t}\right\} .
$$

There are various ways to model short term interest rate $r_{t}$. In the so called single factor models it is modeled as a solution of a certain one-dimensional stochastic differential equation with one-dimensional Brownian motion as a single source of uncertainty. As an example of such models, Vasicek's model can serve in which

$$
d r_{t}=\left(a-b r_{t}\right) d t+\sigma d W_{t},
$$

or Cox-Ingersoll-Ross model (CIR) with

$$
d r_{t}=\left(a-b r_{t}\right) d t+\sigma \sqrt{r_{t}} d W_{t} .
$$

We refer to [30] for more details. An alternative approach to term structure modeling is based on the so called Heath-Jarrow-Morton (HJM) methodology. We assume that the instantaneous forward rate is of the form

$$
f(t, T)=f(0, T)+\int_{0}^{t} \alpha(u, T) d u+\int_{0}^{t} \sigma(u, T) d W_{u} .
$$

Consequently we can consider two kinds of martingale measures:

- martingale measure forward $\hat{P}$, which is equivalent to $P$ and under $\hat{P}, \frac{B(t, T)}{B\left(t, T^{*}\right)}$ is a martingale for $T \leq T^{*}$;

- martingale measure spot $P^{*}$, which is an equivalent to $P$ measure such that $\frac{B(t, T)}{B_{t}}$ is a $P^{*}$ martingale.

The absence or arbitrage corresponds to the existence of martingale forward or spot measures (for details see [30]). In the case of bonds, similarly to the case of defaultable contingent claims, there is a problem to study pricing of financial derivatives, i.e. options based on bonds with the use of stochastic control. Therefore methods preferable in these cases are based on the martingale techniques. 


\section{Portfolio Analysis}

Portfolio analysis is an important area of mathematics of finance. On the other hand this is a part of stochastic control theory. We shall consider two approaches: a static one in which we optimize our portfolio with respect to a certain functional on a one step horizon and a dynamic in which we have to find optimal portfolio for a longer time horizon.

\subsection{Static Portfolios}

Static portfolio is closely related to the so called Markowitz theory. Harry Markowitz (a Nobel prize winner in 1990) was the first economist who admitted the importance to study risk in portfolio analysis (see [28]) considered as a variance of the portfolio growth. Consider a discrete time asset model with prices such that its random rate of return

$$
\zeta_{i}:=\frac{S_{i}(1)-S_{i}(0)}{S_{i}(0)} .
$$

Given portfolio $\pi=\left(\pi_{1}, \pi_{2}, \ldots, \pi_{d}\right)$ representing portions of the wealth process invested in assets and wealth process $V(0)$ at time 0 , under prices $S(1)$, the wealth process $V(1)$ is such that the portfolio rate of return $R^{\pi}$ is of the form

$$
R^{\pi}:=\frac{V^{\pi}(1)-V(0)}{V(0)}=\pi \cdot \zeta,
$$

with - standing for scalar product. We are interested in maximization of the expected portfolio rate of return. On the other hand following Markowitz we would like to minimize the risk considered as the variance of the portfolio rate of return. One can notice that

$$
\operatorname{Var}(R(\pi))=\pi^{*} \Sigma \pi
$$

where $*$ stands for the transpose of the vector $\pi$ and $\Sigma$ is the covariance matrix of the random vector $\zeta$.

Consider now the so called Markowitz order $\succeq$ : we say that portfolio rate of return $R^{\pi}$ is better than $R^{\pi^{\prime}}$ or in other words, the strategy $\pi$ is better than $\pi^{\prime}$, we write $R^{\pi} \succeq R^{\pi^{\prime}}$, whenever $E\left(R^{\pi}\right) \geq E\left(R^{\pi^{\prime}}\right)$ and $\operatorname{Var}\left(R^{\pi}\right) \leq \operatorname{Var}\left(R^{\pi^{\prime}}\right)$.

The maximal elements in this order form a so called efficient frontier on the plane $\left(E R^{\pi}, \operatorname{Var}\left(R^{\pi}\right)\right)$. To determine efficient frontier we maximize $\operatorname{Var}\left(R^{\pi}\right)$ for fixed value of $E\left(R^{\pi}\right)$. This is a quadratic optimization problem with linear constraints. If we admit the so called short selling i.e. allow elements of $\pi$ to be negative, such problem can be solved using standard Lagrange multiplier method (see [29] or [42]). There is a number of problems in implementation of the Markowitz theory. We need an estimate of the

- expected returns $E\left\{\zeta_{i}\right\}$

- covariance matrix $\operatorname{Cov}(\zeta)=E\left\{(\zeta-E \zeta)(\zeta-E \zeta)^{T}\right\}$.

Although the covariance matrix can be well estimated based on historical data there is a problem with the estimation of the expected returns. To overcome this we use a 
Black-Litterman (1990) approach based on Bayesian estimation and forecasted returns (see [3]). Variance of the portfolio as a measure of risk does not seem to be satisfactory. It penalizes in the same way positive events, when portfolio rate of return is above the expected value and negative events, when it is below the expected value. The following measure called value at risk $\operatorname{VaR}_{\alpha}(R(\pi))$ was introduced to banking practice

$$
\operatorname{VaR}_{\alpha}(R(\pi))=\inf \{x: P\{R(\pi)+x \leq 0\} \leq \alpha\} .
$$

This is the smallest value which added to the portfolio rate of return allows nonpositive rate of return with probability below fixed level $\alpha$. Although value at risk is very popular, still it has a number of deficiencies. First of all it measures only probability not the size of inconvenient for us situation. Therefore as a better measure of risk $C V a R_{\alpha}$, i.e. conditional VaR is considered called also expected shortfall

$$
C \operatorname{VaR}_{\alpha}(R(\pi))=E\left\{-R(\pi) \mid R(\pi)+\operatorname{VaR}_{\alpha}(R(\pi)) \leq 0\right\},
$$

which is the expected value of $-R(\pi)$, given that $R(\pi)+\operatorname{Va} R_{\alpha}(R(\pi))$ is nonpositive.

To evaluate $\operatorname{VaR}$ or $C V a R$ we need to know a bit more about the distribution of the random rate of return $\zeta$. Typical assumption that it is multinomial normal is not sufficient, since in practice the densities of such random variables have fat tails. To be more precise one can quote the result of Fergusson and Platen [14] saying that daily logreturns of the world stock market portfolio in 34 different currency denominations form t-Student distribution with 3.94 degrees of freedom. The proper class to study seems to be elliptical distributions introduced in [24]. We say that a $d$-dimensional random vector $X$ is elliptic, whenever its density is of the form

$$
f_{X}(x)=c_{d}|D|^{-\frac{1}{2}} g_{d}\left(\frac{1}{2}(x-\mu)^{T} D^{-1}(x-\mu)\right),
$$

with suitable constant $c_{d}$, positive definite matrix $D$, its determinant $|D|$, vector $\mu$, and a function $g_{d}$. This class includes: normal, t-Student, Laplace, and logistic distributions and is stable under linear transformations. Using a certain standardization procedure we can calculate $\operatorname{VaR}$ and $C V a R$ for elliptically distributed vector $\zeta$ (see [27]). Although computation of the efficient frontier with $\operatorname{VaR}$ or $C V a R$ as risk functions is more complicated, for elliptically distributed $\zeta$ this is a numerically feasible problem.

\subsection{Dynamic Portfolios}

The aspect of risk is also important in the dynamic case. We shall now consider another important optimization problem in mathematics of finance. We are interested to maximize a satisfaction measure as an expected value of a certain utility function of the wealth process. By utility function we mean a concave, increasing function $U$. A class of HARA (hyperbolic absolute risk aversion) utility functions consisting of $U(x)=x^{\alpha}$ with $\alpha \in[0,1)$ and $U(x)=\ln x$ can serve as an example. We maximize terminal utility

$$
E\left\{B_{T}^{-1} U(V(T))\right\},
$$

or a functional with consumption $(c(t))$ 


$$
E\left\{\sum_{t=0}^{T-1} B_{t}^{-1} U_{1}(c(t))+B_{T}^{-1} U_{2}(V(T))\right\},
$$

where $U_{1}, U_{2}$ are utility functions, or we look for growth optimal portfolios (GOP) (see [35]):

$$
E\{\ln (V(T))\} \text {. }
$$

Maximization of the cost functionals (25)-(27) is a stochastic control problem, which in discrete time case can be solved using dynamic programming methods. The continuous time case strongly depends on the form of model for asset prices. The following three approaches can be considered

- solving Hamilton-Jacobi-Bellman equation,

- using convex analysis (duality approach),

- using stochastic maximum principle and BSDEs.

In the next subsections we sketch the main features of these approaches.

\subsubsection{Hamilton-Jacobi-Bellman Equation}

Let

$$
w(t, v, z)=\sup _{\pi}\left\{e^{-\int_{t}^{T} r_{u} d u} U\left(V^{\pi, v}(T)\right)\right\}
$$

be the value function of the terminal utility maximization. The wealth process dynamics under (2) is of the form

$$
d V^{\pi, v}(t)=V^{\pi, v} \pi(t) \cdot \frac{d S(t)}{S(t-)}+V^{\pi, v} \pi_{0}(t) r_{t} d t .
$$

The corresponding Hamilton-Jacobi-Bellman equation is of the form

$$
0=\sup _{\pi}\left[\frac{\partial w}{\partial t}(t, v, z)-r_{t} w(t, v, z)+\mathscr{L}^{\pi, v} w(t, v, z)\right],
$$

with boundary condition $w(T, v, z)=U(v)$, and $\mathscr{L}^{\pi, v}$ being the generator of the pair $\left(V^{\pi, v}, z(t)\right)$. Main problem we have is that the value function (28) is not in the domain of the generator $\mathscr{L}$. Typical verification theorem says that, if we are given a solution to Hamilton-Jacobi-Bellman equation (30), then it coincides with the value function (28). One can extend the notion of solution to (30) introducing the so called viscosity solutions and show that the value function $w$ is a solution to (30) in this sense (for details see [15]).

\subsubsection{Martingale - Convex Analysis Approach}

Consider now a terminal utility maximization problem (25), assuming for simplicity $r_{t}=0$ for $t \geq 0$ (i.e. $B_{t}=1$ ) and that there are no economic factors $(z(t))$ in the asset dynamics. We assume furthermore that the market is complete and the wealth process $\left\{V^{\pi, v}(t)\right\}$ is a martingale under $d Q=\Lambda_{T} d P$, which is a given martingale measure. Clearly

$$
E^{Q}\left\{V^{\pi, v}\right\}=v
$$


Let $\mathscr{H}(v)$ be the family of nonnegative random variables $H$ such that $E\left\{\Lambda_{T} H\right\}=v$. Consider the following static optimization problem:

$$
w(t, v)=\sup _{H \in \mathscr{H}(v)} E\{U(H)\}
$$

We want to find the solution $H^{*}$ to the static problem and the strategy $\pi^{*}$ such that $V^{\pi^{*}, v}(T)=H^{*}$. Assume additionally that the utility function $U$ satisfies the so called Inada conditions: $U^{\prime}(0)=\infty$ and $U^{\prime}(\infty)=0$. Let $I=\left(U^{\prime}\right)^{-1}$. Then

$$
\sup _{v}[U(v)-v y]=U(I(y))-y I(y) .
$$

Using (33) we therefore have

$$
E\{U(H)\} \leq E\left\{U\left(I\left(y \Lambda_{T}\right)\right\}-y\left(E\left[\Lambda_{T} I\left(y \Lambda_{T}\right)\right]-v\right) .\right.
$$

If we choose $y^{*}$ such that $E\left[\Lambda_{T} I\left(y^{*} \Lambda_{T}\right)\right]=v$, we obtain that

$$
E\{U(H)\} \leq E\left\{U\left(I\left(y^{*} \Lambda_{T}\right)\right\} .\right.
$$

Let $H^{*}=I\left(y^{*} \Lambda_{T}\right)$. One can show that $H^{*} \in \mathscr{H}(v)$. Consequently we have that $w(t, v)=$ $E\left\{U\left(H^{*}\right)\right\}$ and $H^{*}$ is a solution to the static problem (32). The optimal strategy $\pi^{*}$ is determined from the martingale representation theorem as a strategy such that $V^{\pi^{*}, v}(T)=$ $H^{*}$. For details see [23].

\subsubsection{Stochastic Maximum Principle}

We now consider another approach to terminal utility maximization (25). Let $S_{i}(t)$ be a solution to the following stochastic differential equation

$$
d S(t)=S(t-)\left(\tilde{a}(t) d t+\sigma(t) d B(t)+\int_{R} \eta(t, x) \bar{N}(d t, d x)\right),
$$

where $\bar{N}$ is the Poisson random measure $N$ for large jumps (taking values outside of the unit ball) and coincides with the compensated Poisson measure $d \tilde{N}_{j}=d N_{j}-d \lambda_{j} d t$ for small jumps (with values from the unit ball). Under the strategy $\pi$ the wealth process $V^{\pi, v}$ is a solution to the equation

$$
\begin{aligned}
V^{\pi, v}(t) & =V^{\pi, v}(t-)\left(\pi_{0}(t) r_{t}+\left(1-\pi_{0}(t)\right) \pi(t) \cdot \tilde{a}(t) d t\right. \\
& +\left(1-\pi_{0}(t)\right)(\pi \cdot \sigma(t) d B(t)+\eta(t, x) \bar{N}(d t, d x)) .
\end{aligned}
$$

We define the Hamiltonian of the form

$$
\begin{aligned}
& M(t, v, \pi, p, q, y)=v\left(\pi_{0} \cdot r_{t}+\left(1-\pi_{0}\right) \pi \cdot \tilde{a}(t)\right) \cdot p+\left(1-\pi_{0}\right) \operatorname{vtr}\left((\pi I \sigma(t))^{T} q\right) \\
& +\left(1-\pi_{0}\right) v \int_{R^{d}}\left\{\sum_{j=1}^{d}\left(\eta_{j}(t, x) y_{j}(t, x)\right)+(\eta(t, x) p+v y)(I-\operatorname{Diag}(\chi))\right\} d \lambda(x)
\end{aligned}
$$

where $I$ is the identity matrix and Diag $\chi$ is the diagonal matrix with $\chi_{j}$ entries such that $\chi_{j}=0$ for large jumps and $\chi_{j}=1$ for small jumps. Consider now the so called adjoint 
equation for $(v, \pi)$, which is backward stochastic differential equation (BSDE) of the form

$$
\begin{aligned}
d p(t) & =-D_{v} M\left(t, V(t), \pi(t), p_{t}, q_{t}, y(t)\right) d t+q_{t} d B_{t} \\
& +\int_{R^{d}} y(t-, x) \bar{N}(d t, d x),
\end{aligned}
$$

with terminal condition $p(T)=D_{v} U(V(T))$ and $D_{v}$ standing for the derivative with respect to $v$. By such solution we mean the triple $(p, q, y)$ for which (38) holds. We have (see [19] and [32]).

Theorem 6. (Stochastic maximum principle): If $(\hat{\pi}, \hat{V})$ is an admissible pair consisting of the strategy $\hat{\pi}$ and corresponding solution to (36) (we assume that there is a solution $\hat{V}$ for such $\hat{\pi})$ and for the triple $(\hat{p}, \hat{q}, \hat{y})$ being the solution to BSDE (38) we have

$$
M(t, \hat{V}(t), \hat{\pi}(t), \hat{p}(t), \hat{q}(t), \hat{y}(t))=\sup _{\pi} M(t, \hat{V}(t), \pi, \hat{p}(t), \hat{q}(t), \hat{y}(t)),
$$

for all $t \in[0, T]$ and $\hat{M}(v):=\sup _{\pi} M(t, v, \pi, \hat{p}(t), \hat{q}(t), \hat{y}(t))$ is concave in $v$, then under some regularity properties $(\hat{\pi}, \hat{V})$ is an optimal pair, i.e. $\hat{\pi}$ is an optimal control while $\hat{V}$ is the optimal wealth process.

\subsection{Long Time Portfolio Functionals}

In an independent section we consider a family of long time growth optimal portfolios (GOP). An infinite horizon analog of (27) is risk neutral GOP with the cost functional (introduced by Kelly in [25])

$$
\liminf _{T \rightarrow \infty} \frac{1}{T} E\{\ln (V(T))\}
$$

An alternative is to study risk sensitive GOP (see [13140]) with the cost functional

$$
\limsup _{T \rightarrow \infty} \frac{1}{\gamma T} \ln E\left\{(V(T))^{\gamma}\right\}
$$

where $\gamma<0$ is the so called risk factor. Such cost function is motivated by the Taylor expansion of the function $F(\gamma)=\ln E\left[e^{\gamma Y}\right]$. Namely, we have $F^{\prime}(0)=E Y, F^{\prime \prime}(0)=$ $\operatorname{Var}(Y)$ and

$$
\frac{1}{\gamma} F(\gamma) \approx E Y+\frac{1}{2} \gamma \operatorname{Var}(Y)
$$

Consequently, maximizing risk sensitive cost functional (41) we maximize the long run expected growth rate diminished by its variance (which is a measure of risk) with weight $-\gamma$. Risk sensitive GOP is a difficult problem, since we have to study a multiplicative cost functional. The risk factor $\gamma$ is negative, however the theory with positive $\gamma$ can be considered as a dual problem to certain long time maximization of the portfolio growth over a given benchmark (see [34] and [41]). Another important feature of the cost functional (41) is that it asymptotically, as $\gamma \rightarrow 0$ approximates risk neutral functional (40). 
The same property can be proved for the optimal values of these functionals (see [12]). More generally, it can be shown (under certain assumptions) that risk neutral nearly optimal strategy is also nearly optimal for risk sensitive cost functional with risk factor $\gamma$ close to 0 . The study of the cost functionals (40) and (41) becomes even harder when we consider proportional, or fixed plus proportional transaction costs. From stochastic control point of view to study risk neutral GOP we have to solve additive Bellman equation, while in the case of risk sensitive GOP we have to solve multiplicative Bellman equations. If we additionally assume fixed costs our strategies are of impulse form (see [33]).

\section{References}

1. Bielecki, T.R., Pliska, S.: Risk sensitive dynamic asset management. JAMO 39, 337-360 (1999)

2. Bielecki, T.R., Rutkowski, M.: Credit Risk: Modelling, Valuation and Hedging. Springer, Heidelberg (2002)

3. Black, F., Litterman, R.: Global portfolio optimization. Financial Analysis J. 48, 28-43 (1992)

4. Black, F., Scholes, M.: The valuation of option contracts and a test of market efficiency. J. Finance 27, 399-417 (1972)

5. Bouchard, B.: No-arbitrage in discrete-time markets with proportional transaction costs and general information structure. Finance Stoch. 10, 276-297 (2006)

6. Campi, L., Schachermayer, W.: A super-replication theorem in Kabanov's model of transaction costs. Finance Stoch. 10, 579-596 (2006)

7. Çetin, U., Jarrow, R., Protter, P.: Liquidity risk and arbitrage pricing theory. Finance Stoch. 8, 311-341 (2004)

8. Çetin, U., Rogers, L.C.G.: Modeling liquidity effects in discrete time. Math. Finance 17, 15-29 (2007)

9. Cox, J.C., Ross, S.A., Rubinstein, M.: Option pricing: a simplified approach. J. Finan. Econom. 7, 229-263 (1979)

10. Dalang, R.C., Morton, A., Willinger, W.: Equivalent martingale measures and no-arbitrage in stochastic securities market model. Stochastics and Stochastics Rep. 29, 185-201 (1990)

11. Delbaen, F., Schachermayer, W.: A general version of the fundamental theorem of asset pricing. Math. Ann. 300, 463-520 (1994)

12. Di Masi, G.B., Stettner, Ł.: Remarks on risk neutral and risk sensitive portfolio optimization. In: Kabanov, Y., Liptser, R., Stoyanov, J. (eds.) From Stochastic Calculus to Mathematical Finance. The Shiryaev Festschrift, pp. 211-226. Springer, Heidelberg (2006)

13. El Karoui, N., Peng, S., Quenez, M.C.: Backward stochastic differential equations in finance. Math. Finance 7, 1-71 (1997)

14. Fegusson, K., Platen, E.: On the distributional characterization of $\log$ returns of a world stock index. Appl. Math. Finance 13, 19-38 (2006)

15. Fleming, W.H., Soner, H.M.: Controlled Markov Processes and Viscosity Solutions. Springer, Heidelberg (1992)

16. Föllmer, H., Kabanov, Y.M.: Optional decomposition and Lagrange multipliers. Finance Stoch. 2, 69-81 (1998)

17. Föllmer, H., Leukert, P.: Quantile hedging. Finance Stoch. 3, 1-25 (1997)

18. Föllmer, H., Schied, A.: Stochastic Finance. An Introduction in Discrete Time. de Gruyter (2002)

19. Framstad, N.C., Oksendal, B., Sulem, A.: Sufficient stochastic maximum principle for the optimal control of jump diffusions and applications to finance. JOTA 121, 77-98 (2004) 
20. Kabanov, Y.M., Kramkov, D.O.: No-arbitrage and equivalent martingale measures: an elementary proof of the Harrison-Pliska theorem. Theory Probab. Appl. 39, 523-527 (1994)

21. Kabanov, Y.M., Rasonyi, M., Stricker, C.: On the closedness of sums of convex cones in $L^{0}$ and the robust no-arbitrage property. Finance Stoch. 7, 403-411 (2003)

22. Kabanov, Y.M., Stricker, C.: A teacher's note on no-arbitrage criteria. In: Sem. Probab. 35. Lecture Notes in Math., vol. 1755, pp. 149-152. Springer, Heidelberg (2001)

23. Karatzas, I.: Lectures on the Mathematics of Finance. AMS (1996)

24. Kelker, D.: Distribution theory of spherical distributions and location-scale parameter generalization. Sankhya 32, 419-430 (1970)

25. Kelly, J.L.: A New Interpretation of Information Rate. Bell System Technical Journal 35, 917-926 (1956)

26. Kociński, M.: Hedging in the CRR model under concave transaction costs. Demonstratio Math. 34, 497-512 (2001)

27. Landsman, Z., Valdez, E.A.: Tail Conditional Expectations for Elliptical Distributions. North American Actuarial Journal 7, 55-71 (2003)

28. Markowitz, H.: Portfolio selection. J. Finance 7, 77-91 (1952)

29. Markowitz, H.: Portfolio Selection Efficient Diversification of Investments. Wiley, Chichester (1959)

30. Musiela, M., Rutkowski, M.: Martingale Modelling in Financial Modelling. Springer, Heidelberg (2005)

31. Nagai, H.: Optimal strategies for risk sensitve portfolio opimization problems for a general factor models. SIAM J. Conrol Opim. 41, 1179-1800 (2003)

32. Oksendal, B., Sulem, A.: Applied Stochastic Control of Jump Diffusions. Springer, Heidelberg (2005)

33. Palczewski, J., Stettner, L.: Maximization of the portfolio growth rate under fixed and proportional transaction costs. Communications in Information and Systems 7, 31-58 (2007)

34. Pham, H.: A Large Deviations Approach to Optimal Long Term Investment. Finance Stoch. 7, 169-195 (2003)

35. Platen, E., Heath, D.: A Benchmark Approach to Quantitative Finance. Springer, Heidelberg (2006)

36. Pliska, S.R.: Introduction to Mathematical Finance. Dicrete Time Models. Blackwell, Malden (1997)

37. Protter, P.E.: Stochastic Integration and Differential Equations. Springer, Heidelberg (2004)

38. Shiryaev, A.N.: Essentials of Stochastic Finance. Facts, Models, Theory. World Scientific, Singapore (2001)

39. Stettner, L.: Discrete time markets with transaction costs. In: Yong, J. (ed.) Recent developments in mathematical finance, pp. 168-180. World Sci. Publ., River Edge (2002)

40. Stettner, L.: Risk sensitive portfolio optimization. Math. Meth. Oper. Res. 50, 463-474 (1999)

41. Stettner, L.: Duality and Risk Sensitvie Portfolio Optimization. Contemporary Mathematics 351, 333-347 (2004)

42. Szegö, G.P.: Portfolio Theory with Application to Bank Asset Management. Academic Press, London (1980) 